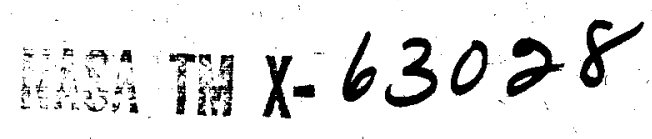

\section{IMPROVEMENT OF MEAN ORBITAL ELEMENTS FOR VINTI'S SPHEROIDAL SATELLITE THEORY}

\section{HAR VEY, WALDEN}

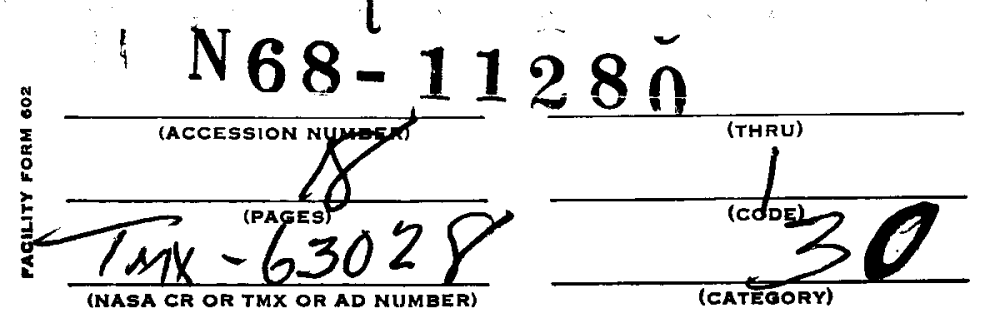

\section{SEPTEMBER 1967}

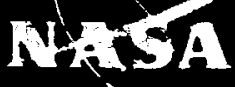

GODDARD SPACE FLIGHT CENTER GREENBELT, MARYLAND 
IMPROVEMENT OF MEAN ORBITAL ELEMENTS

FOR VINTI'S SPHEROIDAL SATELLITE THEORY

Harvey Walden

September 1967

Program Systems Branch

GODDARD SPACE FLIGHT CENTER

Greenbelt, Maryland 


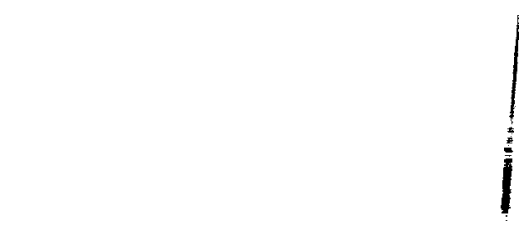

\title{
IMPROVEMENT OF MEAN ORBITAL ELEMENTS \\ FOR VINTI'S SPHEROIDAL SATELLITE THEORY
}

\author{
Harvey Walden
}

\begin{abstract}
A method is proposed for improving the set of mean orbital elements for Vinti's spheroidal satellite theory in the absence of observational data, i.e., when the usual differential method of orbit improvement by a least-squares process is unavailable. An iterative procedure involving the first-order Taylor's series expansion of position and velocity residuals at epoch time is utilized. This process may be used to determine mean orbital elements directly from initial conditions or Keplerian osculating elements, thus eliminating the need for numerical factorization of two quartic polynomials arising in the inversion of the integrals of motion. Alternatively, the method may be applied to find the small corrections to the mean orbital elements following numerical factorization of the quartics, as carried through second order in the oblateness parameter by Vinti. Numerical results are presented indicating both aforementioned applications of the method to actual satellite trajectories.
\end{abstract}


PRECEDING PAGE BLANK NOT FILMED.

TABLE OF CONTENTS

:

$\underline{\text { Page }}$

ABSTRACT ............................... i

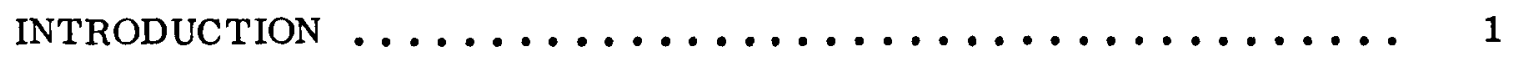

FORMULATION OF THE PROBLEM .................... 3

DIFFERENTIAL COEFFICIENTS .......................... 5

NUMERICAL APPLICATIONS .......................... 10

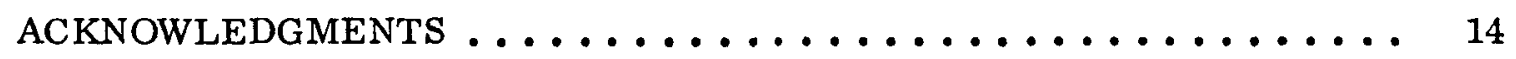

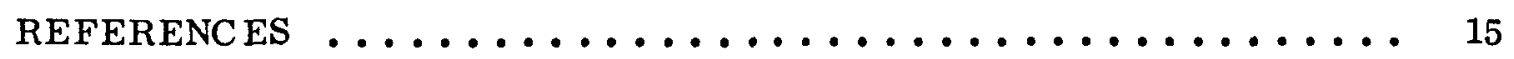


IMPROVEMENT OF MEAN ORBITAL ELEMENTS

FOR VINTI'S SPHEROIDAL SATELLITE THEORY

\section{INTRODUCTION}

The problem of the determination of a set of constants of integration for use in a mathematical theory of orbital motion is ordinarily considered a wholly distinct problem in celestial mechanics from the calculation of motion for a given set of initial conditions or orbital elements. In the spheroidal theory for satellite orbits developed by Vinti (References 1 and 2), a procedure is presented for deriving an accurate reference orbit of any drag-free satellite moving in the gravitational field of an axially symmetrical oblate planet. As applied to artificial satellites of the Earth, the reference orbit takes into account exactly the effects of all zonal harmonic terms in the series expansion of the geopotential through the third, and, in addition, also accounts for the major portion of the fourth zonal harmonic.

In the development of the spheroidal theory, the inversion of the integrals of motion involves the factoring of two quartic polynomials in order to determine the co-ordinates and velocities as functions of time. Vinti proposes (Reference 3) that this factorization be carried out numerically by beginning with a zero-order solution corresponding to Keplerian two-body motion and solving by a method of successive approximations carried through second order in the oblateness parameter. This method allows the constants of the motion originally suggested by Izsak (Reference 4), which permit exact factoring of both quartics, to be found 
correct to second order. It is this set of orbital elements which is used in the equations of the final solution for the co-ordinates and velocities (Reference 5).

In practice, an orbit improvement method is applied to reduce the magnitude of the observational residuals, that is, the numerical discrepancies between positions and velocities predicted by the analytical theory and those actually observed by instruments at the satellite tracking stations. For Vinti's spheroidal satellite theory, a method of orbit improvement through an analytic differential correction has been developed (Reference 6) which minimizes the observational residuals through the principle of least squares, yielding improved values for the orbital elements. Upon convergence of the iterated least-squares fitting process, a mean set bf orbital elements, conditioned by the set of observational data, is produced for use in more accurate predictions. However, the application of this orbit improvement method presupposes the availability of observational data required for the fitting procedure.

Here we wish to propose an alternative method for improving the set of mean elements for Vinti's spheroidal satellite theory in the absence of observational data. An iterative process involving a first-order Taylor's expansion of position and velocity residuals at epoch time is utilized. This procedure may be applied to find the small corrections to the mean orbital elements after the process of numerical factorization of the quartics has been carried through second order. It may also be used to determine mean orbital elements directly from initial conditions (or, equivalently, from Keplerian two-body orbital elements, referred to as osculating elements), thus eliminating the need for numerical factorization. This method, it might be noted, bears certain superficial 
resemblances to an iterative method proposed (Reference 7) for an entirely unrelated general perturbation theory for drag-free satellite orbits, but the differences are substantial. The method to be described enjoys the advantage of being analytic in nature, rather than numerical, so that it is not necessary to rely upon a sufficient criterion for convergence for the iterative process involving partial derivatives which are never evaluated in practice, as is the case in the aforementioned method.

\section{FORMULATION OF THE PROBLEM}

The constants of the motion $q_{i}(i=1,2, \ldots ., 6)$, which are the mean orbital elements for Vinti's spheroidal theory of satellite motion, include the following: the semi-major axis a, the eccentricity e, a parameter $S$ corresponding to the square of the sine of the inclination of the orbital plane to the Equator in Keplerian two-body motion, a parameter $\beta_{1}$ corresponding to the negative of the time of passage through perigee in Keplerian motion, a parameter $\beta_{2}$ corresponding to the argument of perigee in Keplerian motion, and a parameter $\beta_{3}$ corresponding to the right ascension of the ascending node in Keplerian motion. Let us assume that the initial co: Iditions provided are in the form of rectangular inertial position components $X_{0}, Y_{0}, Z_{0}$ and velocity components $\dot{X}_{0}, \dot{Y}_{0}, \dot{Z}_{0}$, given for a particular time $t_{0^{*}}$ (If, in fact, the initial conditions provided are in the form of Keplerian osculating orbital elements, then these may be transformed to inertial position and velocity vector components with the usual two-kody transformation equations.) By the use of the analytical equations of the orbital solution, these six initial components determine the mean orbital elements, through second-order factorization of the quartics. The mean orbital elements are used 
in turn to predict rectangular inertial position and velocity components for arbitrary times $t$. The position and velocity vectors predicted analytically for the epoch time $t_{0}$ will be denoted $X, Y, Z$ and $\dot{X}, \dot{Y}, \dot{Z}$. These components will differ from the initial conditions because of inaccuracies in the numerical factorization process. Of course, if numerical factorization is not utilized, the discrepancies between the set of initial conditions and the predicted position and velocity based upon Keplerian osculating orbital elements will be considerably larger in magnitude.

In any event, it is assumed that the differences in the co-ordinates and veloci-. ties, as well as the required corrections to the orbital elements, are sufficiently small so that their squares and higher powers may be neglected. The residual differences in co-ordinates and velocities may be expressed by a truncated Taylor's series expansion restricted to first powers as

$$
\begin{aligned}
& x_{0}-x=\sum_{i=1}^{6} \frac{\partial x}{\partial q_{i}} \Delta q_{i} \\
& Y_{0}-Y=\sum_{i=1}^{6} \frac{\partial Y}{\partial q_{i}} \Delta q_{i} \\
& . . . . . . . . \\
& \dot{Z}_{0}-\dot{Z}=\sum_{i=1}^{0} \frac{\partial \dot{Z}}{\partial q_{i}} \Delta q_{i}
\end{aligned}
$$


Here the predicted values $X, Y, Z, \dot{X}, \dot{Y}$, and $\dot{Z}$ are viewed as functions of six independent variables, $q_{i}(i=1,2, \ldots, 6)$ which are to be improved by the additive increments $\Delta q_{i}(i=1,2, \ldots, 6)$. The time $t_{0}$ has been omitted as an independent variable because it remains constant throughout. The coefficients in the Taylor's expansion expressing the increment in the co-ordinates and velocities caused by a change in the orbital elements have the form of partial derivatives. These so-called differential coefficients are to be determined analytically from the mathematical equations of motion, as is shown in the following.

\section{DIFFERENTIAL COEFFICIENTS}

There are a total of thirty-six differential coefficients to be determined, but half of these, viz., $\partial X / \partial q_{i}, \partial Y / \partial q_{i}, \partial Z / \partial q_{i}(i=1,2, \ldots, 6)$, have been evaluated previously (Reference 6 ) in the development of an analytic differential correction. From the equations (Reference 5 ) for the velocities $\dot{X}, \dot{Y}$, and $\dot{Z}$, one finds directly that

$$
\begin{aligned}
& \frac{\partial \dot{\mathbf{x}}}{\partial \mathbf{q}_{\mathbf{i}}}=\left(\frac{\rho \dot{\rho}}{\rho^{2}+c^{2}}-\frac{\eta \dot{\eta}}{1-\eta^{2}}\right) \frac{\partial \mathbf{x}}{\partial \mathbf{q}_{\mathbf{i}}} \\
& +\mathbf{x}\left[\left(\rho^{2}+c^{2}\right)^{-1}\left(\rho \frac{\partial \ddot{\rho}}{\partial \mathbf{q}_{\mathbf{i}}}+\dot{\rho} \frac{\partial \rho}{\partial \mathbf{q}_{\mathbf{i}}}\right)-2 \rho^{2} \ddot{\rho}\left(\rho^{2}+c^{2}\right)^{-2} \frac{\partial \rho}{\partial \mathbf{q}_{\mathbf{i}}}\right. \\
& \left.-\left(1-\eta^{2}\right)^{-1}\left(\eta \frac{\partial \dot{\eta}}{\partial \mathbf{q}_{\mathbf{i}}}+\dot{\eta} \frac{\partial \eta}{\partial \mathbf{q}_{\mathbf{i}}}\right)-2 \eta^{2} \dot{\eta}\left(1-\eta^{2}\right)^{-2} \frac{\partial \eta}{\partial \mathbf{q}_{\mathbf{i}}}\right]
\end{aligned}
$$




$$
\begin{aligned}
& -\left(\rho^{2}+c^{2}\right)^{-1}\left(1-\eta^{2}\right)^{-1}\left(a_{3} \frac{\partial Y}{\partial q_{i}}+Y \frac{\partial \alpha_{3}}{\partial q_{i}}\right) \\
& +2 x_{3} Y\left(\rho^{2}+c^{2}\right)^{-2}\left(1-\eta^{2}\right)^{-2}\left[\left(1-\eta^{2}\right) \rho \frac{\partial \rho}{\partial q_{i}}-\left(\rho^{2}+c^{2}\right) \eta \frac{\partial \eta}{\partial q_{i}}\right] \text {, } \\
& \frac{\partial \dot{Y}}{\partial \mathbf{q}_{\mathbf{i}}}=\left(\frac{\rho \dot{\rho}}{\rho^{2}+\mathrm{c}^{2}}-\frac{\eta \ddot{\eta}}{1-\eta^{2}}\right) \frac{\partial \mathbf{Y}}{\partial \mathbf{q}_{\mathrm{i}}} \\
& +\mathbf{Y}\left[\left(\rho^{2}+c^{2}\right)^{-1}\left(\rho \frac{\partial \ddot{\rho}}{\partial \mathbf{q}_{i}}+\dot{\rho} \frac{\partial \rho}{\partial \mathbf{q}_{i}}\right)-2 \rho^{2} \dot{\rho}\left(\rho^{2}+c^{2}\right)^{-2} \frac{\partial \rho}{\partial \mathbf{q}_{i}}\right. \\
& \left.-\left(1-\eta^{2}\right)^{-1}\left(\eta \frac{\partial \dot{\eta}}{\partial \mathbf{q}_{i}}+\dot{\eta} \frac{\partial \eta}{\partial \mathbf{q}_{i}}\right)-2 \eta^{2} \dot{\eta}\left(1-\eta^{2}\right)^{-2} \frac{\partial \eta}{\partial \mathbf{q}_{i}}\right] \\
& +\left(\rho^{2}+c^{2}\right)^{-1}\left(1-\eta^{2}\right)^{-1}\left(a_{3} \frac{\partial \mathbf{x}}{\partial q_{i}}+x \frac{\partial a_{3}}{\partial q_{i}}\right) \\
& -2 \alpha_{3} x\left(\rho^{2}+c^{2}\right)^{-2}\left(1-\eta^{2}\right)^{-2}\left[\left(1-\eta^{2}\right) \rho \frac{\partial \rho}{\partial q_{i}}-\left(\rho^{2}+c^{2}\right) \eta \frac{\partial \eta}{\partial q_{i}}\right] \text {. }
\end{aligned}
$$

and

$$
\frac{\partial \dot{\mathbf{z}}}{\partial \mathbf{q}_{\mathbf{i}}}=\rho \frac{\partial \dot{\eta}}{\partial \mathbf{q}_{\mathbf{i}}}+\dot{\eta} \frac{\partial \rho}{\partial \mathbf{q}_{\mathbf{i}}}+\eta \frac{\partial \dot{\rho}}{\partial \mathbf{q}_{\mathbf{i}}}+\dot{\rho} \frac{\partial \eta}{\partial \mathbf{q}_{\mathbf{i}}}
$$

In the above, the subscript "i" ranges from one to six, corresponding to the orbital elements a, e, $\mathrm{s}, \beta_{1}, \beta_{2}$, and $\beta_{3}$, respectively. All partial derivatives 
in Equations (2), (3), and (4) have been 'expressed explicitly previously (Reference 6$)$, with the exception of those of $\dot{\rho}$ and $\ddot{\eta}$. Note that certain partial derivatives vanish, e.g., $\partial \alpha_{3} / \partial \beta_{1}, \partial \alpha_{3} / \partial \beta_{2}, \partial a_{3} / \partial \beta_{3}, \partial \rho / \partial \beta_{3}$, and $\partial \eta / \partial \beta_{3}$.

The equations for $\ddot{\rho}$ and $\ddot{\eta}$ are slightly modified from the versions originally presented (Reference 5) to the following forms.

$$
\dot{\rho}=\frac{\mathrm{a} \mathrm{e}\left[\left(-2 a_{1}\right)\left(\rho^{2}-2 \mathrm{~b}_{1} \rho+\mathrm{b}_{2}^{2}\right)\right]^{/ / 2}}{\rho^{2}+\mathrm{c}^{2} \eta^{2}} \sin \mathrm{E},
$$

and

$$
\ddot{\eta}=\frac{\mathrm{Q} a_{2}\left(1+\mathrm{c}_{1} \eta-\mathrm{c}_{2} \eta^{2}\right)^{1 / 2}}{\mathrm{u}^{1 / 2}\left(\rho^{2}+\mathrm{c}^{2} \eta^{2}\right)} \cos \psi .
$$

Thus, for $i=1,2,3$ (corresponding to $a, e, S$ ),

$$
\begin{aligned}
& \frac{\partial \dot{\rho}}{\partial \mathbf{q}_{i}}=\frac{\left[\left(-2 a_{1}\right)\left(\partial^{2}-2 b_{1} \rho+b_{2}^{2}\right)\right]^{1 / 2}}{\rho^{2}+c^{2} \eta^{2}}\left[a \text { e cos } E \frac{\partial E}{\partial \mathbf{q}_{i}}\right. \\
& \left.+\left(e \delta_{1 i}+a \delta_{2 i}\right) \sin E\right] \\
& +\dot{\rho}\left[\left(2 a_{1}\right)^{-1} \frac{\partial a_{1}}{\partial \mathbf{q}_{\mathbf{i}}}-2\left(\rho^{2}+c^{2} \eta^{2}\right)^{-1}\left(\rho \frac{\partial \rho}{\partial \mathbf{q}_{\mathbf{i}}}+c^{2} \eta \frac{\partial \eta}{\partial \mathbf{q}_{\mathbf{i}}}\right)\right] \\
& -\frac{2 \text { a e } \alpha_{1} \sin \mathbf{E}}{\left(\rho^{2}+\mathrm{c}^{2} \eta^{2}\right)\left[\left(-2 \alpha_{1}\right)\left(\rho^{2}-2 \mathrm{~b}_{1} \rho+\mathrm{b}_{2}^{2}\right)\right]^{1 / 2}}\left[\left(\rho-\mathrm{b}_{1}\right) \frac{\partial \rho}{\partial \mathbf{q}_{\mathbf{i}}}-\rho \frac{\partial \mathrm{b}_{1}}{\partial \mathbf{q}_{\mathbf{i}}}+\mathrm{b}_{2} \frac{\partial \mathbf{b}_{2}}{\partial \mathbf{q}_{\mathbf{i}}}\right] .
\end{aligned}
$$


In Equation (7), the symbols $\delta_{1 \mathrm{i}}$ and $\delta_{2 \mathrm{i}}$ are Kronecker deltas, defined as follows: $\delta_{i j}=1$ when $i=j$, and $\delta_{i j}=0$ whenever $i \neq j$.

For $\mathrm{i}=4,5$ (corresponding to $\beta_{1}, \beta_{2}$ ), the simpler expression that follows applies.

$$
\begin{aligned}
& \frac{\partial \ddot{\rho}}{\partial \mathbf{q}_{\mathbf{i}}}=\frac{\text { a e }\left[\left(-2 \alpha_{1}\right)\left(\rho^{2}-2 \mathrm{~b}_{1} \rho+\mathrm{b}_{2}^{2}\right)\right]^{1 / 2}}{\rho^{2}+\mathrm{c}^{2} \eta^{2}} \cos \mathbf{E} \frac{\partial \mathbf{E}}{\partial \mathbf{q}_{\mathbf{i}}} \\
& -\frac{2 \dot{\rho}}{\rho^{2}+c^{2} \eta^{2}}\left(\rho \frac{\partial \rho}{\partial \mathbf{q}_{\mathbf{i}}}+c^{2} \eta \frac{\partial \eta}{\partial \mathbf{q}_{\mathbf{i}}}\right) \\
& -\frac{2 \text { a e } \alpha_{1}\left(\rho-b_{1}\right) \sin E}{\left(\rho^{2}+c^{2} \eta^{2}\right)\left[\left(-2 \alpha_{1}\right)\left(\rho^{2}-2 b_{1} \rho+b_{2}^{2}\right)\right]^{1 / 2}} \frac{\partial \rho}{\partial q_{i}} \text {. }
\end{aligned}
$$

The partial derivative of $\dot{\rho}$ with respect to $\beta_{3}$ vanishes.

In similar fashion, for $i=1,2,3$, one finds that

$$
\begin{aligned}
\frac{\partial \dot{\eta}}{\partial \mathbf{q}_{i}} & =\frac{\left(1+C_{1} \eta-C_{2} \eta^{2}\right)^{1 / 2}}{u^{1 / 2}\left(\rho^{2}+c^{2} \eta^{2}\right)}\left[\left(Q \frac{\partial a_{2}}{\partial q_{i}}+\alpha_{2} \frac{\partial Q}{\partial q_{i}}\right) \cos \psi\right. \\
& \left.-Q x_{2} \sin \psi \frac{\partial \psi}{\partial q_{i}}\right]-\dot{\eta}\left[(2 u)^{-1} \frac{\partial u}{\partial q_{i}}+2\left(\rho^{2}+c^{2} \eta^{2}\right)^{-1}\left(\rho \frac{\partial \rho}{\partial q_{i}}+c^{2} \eta \frac{\partial \eta}{\partial q_{i}}\right)\right]
\end{aligned}
$$




$$
\begin{aligned}
& +\frac{\mathrm{Q} a_{2} \cos \psi}{2 u^{1 / 2}\left(\rho^{2}+c^{2} \eta^{2}\right)\left(1+C_{1} \eta-C_{2} \eta^{2}\right)^{1 / 2}}\left[\left(C_{1}-2 \eta C_{2}\right) \frac{\partial \eta}{\partial q_{i}}\right. \\
& \left.+\eta\left(\frac{\partial \mathrm{C}_{1}}{\partial \mathrm{q}_{\mathrm{i}}}-\eta \frac{\partial \mathrm{C}_{2}}{\partial \mathrm{q}_{\mathrm{i}}}\right)\right] \text {, }
\end{aligned}
$$

while for $\mathrm{i}=4,5$, this reduces to

$$
\begin{aligned}
& \frac{\partial \dot{\eta}}{\partial \mathrm{q}_{\mathrm{i}}}=-\frac{\mathrm{Q} x_{2}\left(1+\mathrm{C}_{1} \eta-\mathrm{C}_{2} \eta^{2}\right)^{1 / 2}}{\mathrm{u}^{1 / 2}\left(\rho^{2}+\mathrm{c}^{2} \eta^{2}\right)} \sin \psi \frac{\partial \psi}{\partial \mathrm{q}_{\mathrm{i}}} \\
& -\frac{2 \ddot{\eta}}{\rho^{2}+\mathbf{c}^{2} \eta^{2}}\left(\rho \frac{\partial \rho}{\partial \mathbf{q}_{\mathbf{i}}}+\mathbf{c}^{2} \eta \frac{\partial \eta}{\partial \mathbf{q}_{\mathbf{i}}}\right) \\
& +\frac{\mathrm{Q} \alpha_{2}\left(\mathrm{C}_{1}-2 \eta \mathrm{C}_{2}\right) \cos \psi}{2 \mathrm{u}^{1 / 2}\left(\rho^{2}+\mathrm{c}^{2} \eta^{2}\right)\left(1+\mathrm{C}_{1} \eta-\mathrm{C}_{2} \eta^{2}\right)^{1 / 2}} \frac{\partial \eta}{\partial \mathrm{q}_{\mathrm{i}}} \cdot
\end{aligned}
$$

The partial derivative of $\dot{\eta}$ with respect to $\beta_{3}$ vanishes.

All partial derivatives appearing in Equations (7) through (10) have been determined explicitly (Reference 6), so that the differential coefficients occurring in the first-order Taylor's expansion of the position and velocity residuals may now be evaluated. The simultaneous set (1) of six linear algebraic equations 
admits a solution for the six unknowns, $\Delta q_{i}$, which are used to correct the orbital elements, as follows.

$$
\mathrm{q}_{\mathrm{i}}^{\prime}=\mathrm{q}_{\mathrm{i}}+\Delta \mathrm{q}_{\mathrm{i}}, \quad \mathrm{i}=1,2, \ldots, 6 .
$$

These corrected orbital elements are used to predict analytically a new set of position and velocity components at epoch time and to re-evaluate the differential coefficients in Equation (1). The process is then continued iteratively until the absolute values of the position and velocity residuals at epoch time, $\left|X_{0}-X\right|$, $\left|Y_{0}-Y\right|, \ldots,\left|\dot{Z}_{0}-\dot{Z}\right|$, reach some sufficiently small predetermined values.

\section{NUMERICAL APPLICATIONS}

This iterative method of improving a set of mean elements for Vinti's spheroidal satellite theory without the use of observational data has been applied to actual satellite trajectories. The results of an application to determine mean Izsak orbital elements directly from initial conditions, i.e., without the use of numerical factorization to evaluate the elements to second order, is summarized in Table 1-A. The first column lists the initial conditions for a particular epoch of an artificial satellite orbit in the form of inertial rectangular co-ordinates and their time derivatives. The second column provides the osculating Keplerian orbital elements corresponding to the inttial conditions. These classical Keplerian elements were obtained through use of the two-body equations of motion, considering only the central term in the potential field. The final column displays the converged values for the mean set of Izsak orbital elements obtained after 
three iterations. A measure of the degree of improvement in the orbital elements is provided by Table $1-\mathrm{B}$, which displays the residuals in position and velocity components, using the osculating Keplerian elements initially and then the mean iterated Izsak elements obtained upon convergence.

The results of an application to improve the mean Izsak orbital elements following the use of numerical factorization of the quartics through second order is summarized in Table 2-A. The initial conditions for an artificial satellite orbit are identical to those presented previously. The second column now provides the mean Izsak orbital elements determined by numerical factorization carried through second order in the Earth's oblateness parameter, and the final column gives the converged values for the mean Izsak orbital elements obtained after two iterations. Table 2-B shows the residuals in position and velocity components with use of tine two respective sets of mean Izsak orbital elements.

It is seen that the final iterated set of mean Izsak orbital elements is virtually independent of whether the process of numerical factorization of the quartics is utilized. Hence the iterative method of determining mean elements for Vinti's spheroidal satellite theory presented in this paper may be used as a valid alternative to the procedure of numerical factorization. However, if the latter procedure is applied, then substantial corrections to the orbital elements are not to be expected through use of the iterative method, inasmuch as any such corrections account for errors of third or higher orders. Without use of numerical factorization, the decrease in size of the position and velocity residuals through application of the iterative method is impressive, but even when the factorization process is employed, most residuals experience noteworthy decreases after only two iterations of this method. 
TABLE 1-A

Determination of Mean Izsak Orbital Elements Directly from Initial Conditions

\begin{tabular}{crcc}
\hline Initial Conditions $\mathrm{a} /$ & $\begin{array}{c}\text { Osculating } \\
\text { Keplerian Elements }\end{array}$ & $\begin{array}{c}\text { Mean Iterated } \\
\text { Izsak Elements } /\end{array}$ \\
\hline $\mathbf{x}=+0.86773200$ & $\mathrm{a}=1.7478170$ & $\mathrm{a}=1.7461661$ \\
$\mathrm{y}=+1.0052368$ & $\mathrm{e}=0.23597612$ & $\mathrm{e}=0.23553637$ \\
$\mathbf{z}=-0.14256217$ & $\mathrm{sin}^{2} \mathrm{i}=0.52617614$ & $\mathrm{~S}=0.52593981$ \\
$\ddot{\mathbf{x}}=-0.54766917$ & $-\tau=0.056312786$ & $\beta_{1}=0.052921090$ \\
$\dot{\mathbf{y}}=+0.38465985$ & $\omega=3.2487135$ & $\beta_{2}=3.2503452$ \\
$\dot{z}=-0.69095995$ & $\Omega=-2.3849592$ & $\beta_{3}=-2.3844409$ \\
\hline
\end{tabular}

a/The position components $x, y, z$ are in Earth equatorial radii (e.r.), and the velocity components $\dot{x}, \dot{y}, \dot{z}$ are in e.r./c.u.t., where 1 canonical unit of time (c.u.t.) is equal to 806.823 seconds. The semi-major axis a is in e.r., the time of perigee passage $\tau$ and $\beta_{1}$ are in c.u.t., and the argument of perigee $\omega$, the right ascension of the ascending node $\Omega, \beta_{2}$, and $\beta_{3}$ are in radians.

TABLE 1-B

Magnitude of Residuals in Position and Velocity Components

\begin{tabular}{ccc}
\hline Residual a/ & $\begin{array}{c}\text { Initially } \\
\text { (Keplerian Elements) }\end{array}$ & $\begin{array}{c}\text { Upon Convergence } \\
\text { (after 3 iterations) }\end{array}$ \\
\hline$|\Delta x|$ & 1722.44 & 0.29 \\
$|\Delta y|$ & 2133.78 & 0.38 \\
$|\Delta z|$ & 5425.18 & 0.05 \\
$|\Delta \dot{x}|$ & 95.2453 & 0.0118 \\
$|\Delta \dot{y}|$ & 59.9060 & 0.0471 \\
$|\Delta \dot{z}|$ & 85.3974 & 0.0177 \\
\hline
\end{tabular}

a/ The position residuals are in meters, and the velocity residuals in centimeters per second. 
TABLE 2-A

Improvement of Mean Izsak Orbital Elements After Use of Second-Order Numerical Factorization

\begin{tabular}{lcc}
\hline \hline Initial Conditions $\underline{\text { a/ }}$ & $\begin{array}{c}\text { Numerically Factored } \\
\text { Mean Izsak Elements }\end{array}$ & $\begin{array}{c}\text { Mean Iterated } \\
\text { Izsak Elements }\end{array}$ \\
\hline $\mathbf{x}=+0.86773200$ & $\mathrm{a}=1.7461655$ & $\mathrm{a}=1.7461661$ \\
$\mathrm{y}=+1.0052368$ & $\mathrm{e}=0.23553626$ & $\mathrm{e}=0.23553640$ \\
$\mathbf{z}=-0.14256217$ & $\mathrm{~S}=0.52594027$ & $\mathrm{~S}=0.52593981$ \\
$\dot{\mathbf{x}}=-0.54766917$ & $\beta_{1}=0.052921097$ & $\beta_{1}=0.052921090$ \\
$\dot{y}=+0.38465985$ & $\beta_{2}=3.2503450$ & $\beta_{2}=3.2503453$ \\
$\dot{z}=-0.69095995$ & $\beta_{3}=-2.3844408$ & $\beta_{3}=-2.3844408$ \\
\hline
\end{tabular}

a/ The position components $x, y, z$ are in Earth equatorial radii (e.r.), and the velocity components $\dot{x}, \dot{y}, \dot{z}$ are in e.r./c.u.t., where 1 canonical unit of time (c.u.t.) is equal to 806.823 seconds. The semi-major axis a is in e.r., the parameter $\beta_{1}$ is in c.u.t., and the parameters $\beta_{2}$ and $\beta_{3}$ are in radians.

TABLE 2-B

Magnitude of Residuals in Position and Velocity Components

\begin{tabular}{ccc}
\hline Residual $/$ & $\begin{array}{c}\text { Initially } \\
\text { (Factored Elements) }\end{array}$ & $\begin{array}{c}\text { Upon Convergence } \\
\text { (after 2 iterations) }\end{array}$ \\
\hline$|\Delta \mathbf{x}|$ & 0.0475 & 0.3802 \\
$|\Delta y|$ & 1.9008 & 0.2851 \\
$\mid \Delta z^{\prime}$ & 1.4375 & 0.0233 \\
$|\Delta \dot{x}|$ & 0.2238 & 0.0118 \\
$|\Delta \dot{y}|$ & 0.0294 & 0.0353 \\
$|\Delta \dot{z}|$ & 0.2827 & 0.0353 \\
\hline \hline
\end{tabular}

a/

- The position residuals are in meters, and the velocity residuals in centimeters per second. 


\section{ACKNOWLEDGMENTS}

The author is grateful to N. L. Bonavito of the Mission Trajectory Determination Branch for suggestions proposed in discussions relating to the theoretical aspects of this report and to Stan Watson of the Program Sysitems Branch for assistance in the numerical calculations required. 


\section{REFERENCES}

1. Vinti, John P., "Invariant Properties of the Spheroidal Potential of an Oblate Planet," J. Res. Nat. Bur. Stds. (U.S.), 70 B (Math. and Math. Phys.), No.1, pp. 1-16, January-March, 1966.

2. Vinti, John P., "Inclusion of the Third Zonal Harmonic in an Accurate Reference Orbit of an Artificial Satellite," J. Res. Nat. Bur. Stds. (U.S.), 70 B (Math. and Math. Phys.), No. 1, pp. 17-46, January-March, 1966.

3. Vinti, John P., "Theory of an Accurate Intermediary Orbit for Satellite Astronomy," J. Res. Nat. Bur. Stds. (U.S.), 65 B (Math. and Math. Phys.), No. 3, pp. 169-201, July-September, 1961.

4. Izsak, Imre G., "A Theory of Sa:ellite Motion About an Oblate Planet: A Second-Order Solution of Vinti's Dynamical Problem," Smithsonian Institution Astrophysical Observatory, Research in Space Science Special Report No. 52, November, 1960 .

5. Bonavito, N. L., "Computational Procedure for Vinti's Accurate Reference Orbit with Inclusion of the Third Zonal Harmonic," NASA Technicr.1 Note D-3562, August, 1966 .

6. Walden, Harvey and Watson, Stan, "Differential Corrections Applied to Vinti's Accurate Reference Satellite Orbit with Inclusion of the Third Zonal Harmonic," NASA Technical Note D-4088, September, 1967.

7. Cain. Barbara J., "Determination of Mean Elements for Brouwer's Satellite Theory," Astron. J., 67, No. 6, pp. 391-392, August, 1962. 